\title{
LE FRANÇAIS DU DROIT COMMERCIAL EN CONFRONTATION AVEC LE TCHÈQUE
}

\author{
Janka Priesolová
}

\begin{abstract}
The article investigates a contrastive analysis of professional French and Czech in the framework of IGA project at the University of Economics in Prague, called European Terminology in Economics and Law. After a general lexicological and stylistic characterisation of French used in business law, the stress is put on contrastive analysis of French and Czech business law in the field of law and liguistics with the emphasis on the fact that a reformulation and transfer of a particular content between different linguistic codes requires not only good language and linguistic competence, but also an adequate additional professional competence. Further, the article proposes a particular linguistic presentation of legal vocabulary, which includes also certain thematic micro-systems with terms used in their most common collocations while their language equivalents are determined not only by their position in their own language systems, but also by their position within adequate non-linguistic contexts (i.e. legal systems).

Keywords: contastive linguistics; professional French; legal subsystem of a language; French of business law; linguistic and non-linguistic context.

Résumé : L'article est consacré à l'analyse contrastive du français de spécialité en confrontation avec le tchèque, réalisée dans le cadre du projet scientifique IGA no 13/2010 de l’Université d'économie de Prague et réservé à la Terminologie européenne en économie et en droit. Après avoir caractérisé le français $\mathrm{du}$ droit commercial au niveau lexical et stylistique, l'article se concentre à l'analyse contrastive du droit commercial en français et en tchèque du point de vue juridique et linguistique en mettant l'accent sur le fait que la reformulation et le tranfert de sens entre deux codes linguistiques différents exige non seulement une compétence linguistique mais aussi une compétence professionnelle spécialisée complémentaire. La suite propose une présentation linguistique du lexique juridique contenant des micro-systèmes thématiques où les termes sont étudiés dans leurs collocations les plus courantes et dont les équivalents sont choisis en fonction des places que ces termes occupent dans leurs propres systèmes linguistiques aussi bien que dans des contextes extralinguistiques respectifs (à savoir en droit commercial).
\end{abstract}

Mots-clés : linguistique contrastive ; français de spécialité ; langage juridique ; français du droit commercial ; contexte linguistique et extralinguistique.

\section{Introduction}

Le présent article attire attention sur certains problèmes de linguistique contrastive que nous avons rencontrés lors des travaux sur notre projet scientifique réalisé au sein de l’Université d'économie de Prague concernant La terminologie européenne en économie et en droit. Nous nous sommes concentrés tout particulièrement sur l'analyse du droit commercial. L'objectif de notre projet (de deux ans) auquel participent trois étudiants de master en droit commercial et deux enseignants de français de spécialité, est de com- 
parer les conditions et les environnements juridiques tchèque et français dans lesquels se déroulent les relations commerciales et de signaler les différences dans la détermination et le fonctionnement des institutions françaises et tchèques respectives à travers l'analyse de la terminologie et le lexique spécifiques dans les domaines les plus représentatifs du droit commercial (aussi bien au niveau du droit privé que public). Il s'agit donc non seulement de l'analyse des moyens linguistiques spécifiques de chaque langue, mais aussi de l'analyse de différences extralinguistiques et interculturelles recouvrant les domaines du droit commercial tchèque et français qui se reflètent indéniablement dans chaque langue. En effet, les termes sont souvent étroitement liés à des pratiques sociales et à un environnement culturel différent d'un pays à l'autre. L'appareil terminologique structuré en français et en tchèque devrait faire ressortir les traits spécifiques de chaque langue (étant typologiquement différentes) tout en reflétant les particularités des discours professionnels et des environnements juridiques respectifs.

Dans la première étape de notre projet nous nous sommes concentrés sur l'analyse du cadre juridique général de la vie commerciale, sur les acteurs et les conditions matérielles des activités commerciales (droit de la propriété), sur différents types de sociétés commerciales, les groupements d'intérêt économique, sur le droit de la concurrence, sur les systèmes fiscaux notamment dans le domaine des impôts directs des personnes morales. Il est bien clair que l'analyse et la description des systèmes juridiques sont très étroitement liées au choix de moyens terminologiques appropriés. Nous avons insisté sur le fait que dans la recherche des équivalents terminologiques entre le français et le tchèque, il est indispensable de procéder par l'analyse indépendante des domaines analysés d'après les sources de droit français et tchèque à part.

Dans notre article nous allons tout d'abord caractériser les traits spécifiques de la langue du droit des affaires en français au niveau des discours législatif, juridictionnel et contractuel, pour nous concentrer dans la suite sur l'analyse linguistique du français juridique en confrontation avec le tchèque tout en signalant certains problèmes dans la recherche des équivalents terminologiques entre les deux langues.

\section{Le français du droit commercial}

Une langue de spécialité est un sous-système spécialisé de moyens linguistiques mis au service d'une communication spécialisée. Le langage juridique est donc une compétence communicative qui combine des facteurs linguistiques, socioculturels et juridiques où l'expression linguistique appropriée fait resortir non seulement les particularités d'une communication juridique (au niveaux lexical, sémantique et stylistique), mais aussi ceux de différents ordres juridiques des communautés socio-culturelles respectives.

La langue française du droit commercial (ou du droit des affaires au sens large du terme) représente des traits spécifiques surtout au niveau lexical, mais aussi au niveau syntaxique et stylistique. Le vocabulaire juridique se doit par définition d'être précis pour ne pas faire l'objet d'aucune interprétation divergeante ce qui pourrait avoir des conséquences négatives en cas de contentieux. Il comprend plusieurs types de termes ou d'expressions juridiques. En dehors des termes d'appartenance juridique exclusive (où la monosémantisation intervient déjà au niveau de la dénotation), il y en a aussi ceux qui font partie non seulement de la langue juridique, mais aussi de la langue courante. ${ }^{1}$

\footnotetext{
D’après la catégorisation établie par Cornu 1990.
} 
Les premiers, réservés aux professionnels du droit, ne sont utilisés que dans le contexte juridique avec le sens souvent obscur pour les non initiés (ayant droit, greffier, mandataire-liquidateur, référé commercial, saisine du tribunal de commerce, séquestre, etc.), tandis que les mots à double appartenance, qui sont d'ailleurs plus nombreux, peuvent apparaître dans les contextes différents revêtant aussi des significations différentes au niveau des désignations concrètes. Dans cette catégorie il y a, d'une part, des termes d'appartenance juridique principale qui sont passés dans la langue courante ce qui représente d'ailleurs une sorte d'enrichissement du français à partir du vocabulaire juridique (arbitre, délit, contrat, gage, loi, témoin, magistrat, procédure, procès, sanction, justice, juge, contentieux, etc.). ${ }^{2}$ $\mathrm{D}^{\prime}$ autre part, il y a aussi des mots de la langue courante qui passent dans la langue juridique avec un sens spécifique bien précis (termes d'appartenance juridique secondaire) - minute, gosse (« original et copie certifiée d'un jugement »), commerçant (« celui qui exerce une activité d'entreprise»), murs commerciaux («biens utilisés pour un usage professionnel »), meuble (« un bien corporel pouvant être transporté »), objet du contrat, prix de la chose, etc.

Le vocabulaire du français commercial contient également des archaïsmes parce que le code de commerce français actuel est une reformulation du code de 1807 même si celui-ci est passé par plusieurs remaniements et actualisations (entreprise de manufacture, vente à l'encan, voiturier, voiturage - remplacé par transport seulement en 2004). Une autre caractéristique est le maintien de nombreuses tournures empruntées au latin (ceux qui sont devenus français : récépissé, ratio, quorum..., mais aussi ceux qui enrichissent en latin le discours juridique : dolus bonus, dolus malus, fructus usus, stricto sensu, fraus omnia corrompit - « la fraude corrompt tout », etc.). D’autre part, à la différence du français des affaires, le français juridique comprend très peu d'anglicismes (stock, label, know-how). En ce qui concerne les néologismes ou internationalismes, ceux-ci apparaissent surtout en liaison avec l'harmonisation du droit européen (acquis communautaire, subsidiarité) et ce sont justement ces nouveaux termes d'origine française qui sont adoptés par d'autres langues, y compris le tchèque. De plus, le renouvellement constant du vocabulaire au niveau national résulte aussi de la nécessité de refléter de nouveaux besoins de la vie économique et sociale où le droit est fortement enraciné (procédure de sauvegarde, autoentrepreneur, Autorité de la concurrence, préjudice environnemental, etc.).

En ce qui concerne la grammaire et la syntaxe, le discours législatif est caractérisé par la clarté, précision, concision, étant dépourvu de toute affection ou subjectivité. C'est un discours qui interdit ou autorise quelque chose : disposition graphique du texte avec ses subdivisions en paragraphes, emplois des verbes exprimant différents types de modalité (pouvoir, permettre, obliger, forcer, interdire, prohiber, défendre), articulateurs (du type nonobstant, attendu que, en vertu que, au titre de, sauf si, toutefois), nominalisations, pronoms indéfinis (tout, chaque, aucun, nul), constructions impersonnelles (il y a, il est tenu, il appartiet, il est interdit), usage de la $3^{\mathrm{e}}$ personne du singulier (le débiteur, le vendeur, le liquidateur...), participes présent et passé, emploi de la voie passive (le montant du capital social est fixé par les statuts, il est divisé en parts sociales égales), emplois du présent de l'indicatif et de l'inversion du sujet (figurent au registre les inscriptions et actes...), etc. Tandis que les discours législatif et juridictionnel sont fortement structurés, le discours contractuel

\footnotetext{
2 Ce processus est appelé banalisation lexicale au cas où sa portée est générale (Tomášek : 1984, 1998), ou bien l'individualisation lexicale dans le cas d'un usage professionnel particulier « relaché » ou argotique (Galisson : 1972).
} 
est caractérisé par une grande diversité se rapprochant davantage au langage standard. En effet, les contrats sont en général rédigés en langage courant au nom du principe du « consentement éclairé » des partis.

\section{Analyse contrastive de la langue du droit commercial français et tchèque}

\section{a) Analyse lexico-sémantique et sémiotique}

Le langage juridique est un ensemble de moyens linguistiques spécialisés qui servent à transmettre les informations spécialisées d'ordre juridique. Pour transposer le lexique ou le discours d'une langue à l'autre, il faut non seulement avoir une bonne compétence linguistique dans les deux langues, mais également une compétence extralinguistique (en matière du droit commercial) qui consiste, entre autre, en détérmination ou identification des concepts et notions exprimés par les termes respectifs. ${ }^{3}$

Bien que les moyens lexicaux et grammaticaux contribuent à la désignation dans leurs rapports synergétiques, c'est le lexique qui constitue la base principale dans l'acquisition du français de spécialité. Cependant on ne conçoit pas le lexique comme un répertoire terminologique au sens étroit du terme, mais comme un système complexe d'unités onomatologiques (simples ou complexes) entre lesquelles il existe toute une série de rapports morpho-syntaxiques, lexico-sémantiques et même thématiques. Une bonne acquisition de ces unités est étroitement liée avec l'analyse de leurs rapports formelles et sémantiques aussi bien sur l'axe paradigmatique (dérivations, synonymes, antonymes) que sur l'axe syntagmatique (possibilités combinatoires, collocations). Ainsi le lexique ou le "vocabulaire » juridique contient non seulement les termes juridiques au sens étroit du terme (loi, droit, délit / zákon, právo, trestný čin), mais aussi les combinaisons de mots plus ou moins figées représentant l'usage et l'emploi de ces termes dans le discours (adopter une loi - prijimout zákon, prononcer un jugement - vynést rozsudek, acte arbitraire - svévolný čin, acte notarié - notářská listina, acte de commerce - obchodní záležitost...). Il ne faut pas non plus oublier des phrases-modèles (Le présent contrat est conclu pour une période de...).

J'aimerais signaler quelques problèmes relatifs à la recherche des équivalents entre les termes français et tchèques que nous avons rencontrés lors de l'analyse du lexique du droit des affaires au sens large du terme :

* La même réalité extralinguistique désignée par des unités onomatologiques simples ou complexes - termes précis dans les deux langues ayant une équivalence sémantique univoque : impôt - daň, droits de douane - clo, société commerciale - obchodní společnost, concurrence - hospodářská soutěž.

* La même réalité extralinguistique formalisée d'une manière différente au niveau de la dénotation : l'institution tchèque finanční úr̆ad ("bureau des finances ») a pour équivalents français les termes tels que administration fiscale, fisc, recette des impôts (qui corresponderaient plutôt à "daňový » úr̆ad); à l'expression obchodní akademie correspond lycée technologique (STG - série sciences et technologie de la gestion); zaměstnanec a OSVČ sont traduits comme salarié et (travailleur) indépendant, soudce konkurzní podstaty ou bien plutôt insolvenční správce est administrateur judiciaire (qui a remplacé à son tour l'ancien syndic

\footnotetext{
3 « Connaître le vocabulaire juridique c'est posséder les clés de la matière, avoir accès aux classifications, aux raisonnements, aux contreverses » (Cornu 1990).
} 
de la faillite). Si l'on traduisait ces notions littéralement (mot à mot), elles perderaient leur sens et seraient incompréhensibles dans la langue d'arrivée (LA).

* La réalité extralinguistique différente (différents contextes socio-économiques, administratifs ou juridiques) - dans la LA il n'y a pas d'équivalent précis, certaines notions de la langue de départ (LD) ne sont pas formalisées dans la LA, il faut donc les décrire ou paraphraser pour les rendre compréhensibles : officier ministériel - veřejná osoba (notár̆, exekutor, licitátor ve Francii), juge consulaire - soudce obchodného soudu ve Francii, foyer fiscal - daňová domácnost ve Francii, GIE - hospodářské zájmové sdružení(F), différents types d'impôts ou de sociétés commerciales qui n'existent pas en République tchèque et vice versa.

$C^{\prime}$ est également le cas des noms des institutions qu'il est préférable de garder dans leur forme originale en proposant le nom d'une institution équivalente (si elle existe) dans la LA. En plus, dans ce cas il ne s'agit souvent que d' « équivalents » approximatifs étant donné la possibilité des compétences différentes de ces institutions dans les systèmes socio-culturels respectifs : INSEE - statistický úr̆ad ve Francii à côté du terme neutre Office des statistiques, Pôle d'emploi, qui a remplacé ANPE (úřad práce ve Francii) à côté de l'équivalent non marqué Bureau du travail. Registre des métiers français trouve son équivalent juridique tchèque dans Živnostenský úřad. DGCCRF ("Generální ředitelství pro hospodářskou soutěž, spotřebu a potírání podvodů ») et Autorité de la concurrence (pour les abus de la concurrence) trouveront leur correspondant institutionnel tchèque dans Úr̆ad pro hospodářskou soutěž ("Office pour la protection de la concurrence »).

La langue est le moyen privilégié qui sert à la transmission d'une information spécialisée (juridique dans notre cas). Le processus de traduction n'est pas seulement une transmission de code linguistique donné, mais surtout une sorte de réexpression de sens par des moyens linguistiques appropriés. Pour traduire un terme d'une langue à l'autre, il faut donc réaliser d'abord une traduction intrasémiotique pour établir le sens précis (« le(s) dénoté(s) ») de la réalité désignée par le signe linguistique («le dénotant / le désignant ») dans la langue de départ, pour passer après à une traduction intersémiotique qui permet d'attribuer aux "dénotés » du code juridique de la langue d'arrivée une forme adécuate (« désignant») en fonction de ses propres codes juridique et linguistique. ${ }^{4}$

Prenons comme exemple actes de commerce, commerçant - podnikání (činnost), podnikatel. En effet, en droit commercial le commerçant (dont le sens économique corrrespond en tchèque à obchodnik) a pour l'équivalent plutôt podnikatel (« personne physique ou morale qui effectue des actes de commerce et en fait sa profession, agissant en son nom et pour son propre compte »), mais ces termes sont construits différemment au sein des communautés juridiques respectives. Cependant le sens ou la dénotation du terme tchèque podnikatel est beaucoup plus large parce qu'il recouvre également les professions libérales, les exploitants agricoles et les artisans qui n'ont pas de statut de commerçant en France. Le terme tchèque žionostník (« osoba podnikající na základě živnostenského oprávnění ») n'a pas non plus un équivalent univoque. Cela peut être aussi bien un artisan qu'un commerçant ou bien un entrepreneur individuel, ainsi qu'un auto-entrepreneur (" petit entrepreneur » dans le sens du droit européen) qui peut exercer une activité indépendante supplémentaire sous une forme juridique simplifiée. En ce qui concerne le terme tchèque podnik il ne peut être traduit par entreprise que dans le sens d'une unité économique au sens étroit du terme. En revanche, si on prend en compte son entité juridique distincte des éléments qui le composent (« podnik jako předmět vlastnickoprávních vztahů » - 55

4 Cf. Knapp 1995, Tomášek 1998. 
$\mathrm{du}$ code de commerce tchèque), on emploi un terme difficilement traduisible fonds de commerce (" ensemble des éléments mobiliers corporels et incorporels »). En droit français on distingue effets de commerce - směnky a šeky et valeurs mobilières - cenné papíry (akcie a dluhopisy), émises en masse qui sont quelquefois appelées en tchèque par un faux ami " efekty ». En droit français une entreprise qui ne peut plus accomplir ses engagements a une obligation de déposer le bilan ("ohlásit úpadek ») ce qui correspond en droit tchèque à la proposition de prohlásit konkurs. Le tribunal de commerce français peut décider d'un règlement / redressement judiciaire ("soudní narovnání » corresponsant à úpadkové/konkursní ř́zení, remplacé en 2008 par insolvenční rízení) avant de passer à la liquidation judiciaire - soudní likvidace, dražba. Cependant, pour régler les problèmes des entreprises en difficulté, le droit français a institué en 2006 la procédure de sauvegarde (« ochranné řízení ») en tant qu'une mesure préventive, suspendant aux entreprises le paiement de leurs dettes précédant l'ouverture de la procédure du redressement judiciaire.

L'analyse des termes est en rapport étroit non seulement avec la prise en compte de leur sens dans le système extralinguistique respectif, mais aussi de celui qui leur est attribué dans le système linguistique donné. En effet la dénotation de termes correspondants dans les deux langues n'est pas forcément identique parce qu'elle est déterminée en fonction des rapports de ce terme avec d'autres termes existant dans le cadre d'un champ sémantique donné. Il y a par exemple différentes expressions françaises pour " sleva ». À côté du terme générique réduction, il en existe d'autres (rabais, remise, escompte, ristourne, solde, promotion) d'après les conditions concrètes dans lesquelles elles sont accordées ; par ailleurs il y a aussi «sleva na dani »-déduction, abattement, allègement fiscal(e) ("odečitatelné položky od daňového základu ») nebo avantage fiscal, réduction fiscale, crédit d'impôt, dégrèvement fiscal, exonération fiscale (různé " úlevy na dani, jejich snížení či osvobození od daně »).

\section{b) Présentation linguistique des unités lexicales}

Notre étude du lexique du droit des affaires est centrée avant tout sur l'analyse complexe de termes clés de certains domaines thématiques (micro-systèmes notionnels) en mettant l'accent sur les possibilités dérivationnelles de ces termes, mais surtout sur l'étude de leurs collocations syntagmatiques aussi bien que de leurs possibilités combinatoires dont les plus importants sont celles avec adjectifs ou verbes (constructions verbonominales de caractère libre ou idiomatique, figé). Leurs constructions les plus fréquentes avec les verbes ou les adjectifs témoignent ainsi souvent d'une combinabilité lexico-sémantique au niveau de la dénotation. D'autre part, ces chaînes de termes en combinaisons syntagmatiques représentent une source de références thématiques supplémentaires de signification économique et juridique tout en apportant une aide précieuse pour un étranger qui n'a pas l'intuition d'un locuteur natif dans la construction d'un discours juridique.

* commerce m - obchodování, podnikání, prodejna (commerçant, commercialisation, commercial, commercer, commercialiser)

+ verbe : faire/stimuler/réglementer/libéraliser le ; gérer/développer/fermer/céder son ; tenir un petit ; stagner/régresser/s'effondrer

+ adjectif ou complément de nom : éléctronique/extérieur/intérieur/équitable/lucratif/indépendant (isolé, associé)/ intégré (F) ; de gros/de détail/en libre service...; chambre de /code del tribunal de/effet de/ livres de/ acte de/fonds de/représentant de $\sim, \ldots$ 
* obchod m - commerce (troc, négoce, affaire, échange, marché) ; magasin, boutique (obchodník, obchodovat, obchodni, obchodovatelný, maloobchod, velkoobchod)

+ verbe : dělat/otevřit si , běhat po $\sim$ ech, je to dobrý ...)

+ adjectif : bezcelní (commerce en franchise douanière), komisní (dépôt-vente), zahraniční (commerce étranger), burzovní (opérations de Bourse), výměnný (troc); spravedlivý (commerce équitable), s narkotiky ( trafic), zóna volného (zone de libre échange), etc.

\section{Conclusion}

Le but de notre projet est de présenter les informations structurées dans le domaine du droit des affaires en présentant un appareil terminologique de base (en fonction des codes linguistiques des deux langues) qui reflèterait les environnements juridiques respectifs tout en mettant l'accent sur l'étude « contextuelle » de termes clés choisis. L'analyse du lexique français pourrait non seulement se matérialiser dans un dictionnaire (français tchèque et tchèque - français), mais elle pourrait également enrichir l'étude des langues de spécialité par un domaine spécifique du droit commercial auquel n'était réservée, jusqu'à présent, qu'une place limitée dans nos dictionnaires juridiques actuels. En effet, les aspects juridiques de la vie commerciale représentent un domaine important qui est en évolution dynamique surtout dans le contexte de l'intégration européenne et de celui de la globalisation économique. Il n'est pas non plus négligeable dans les conditions de notre économie marquée par les investissements étrangers croissants, par l'implantation des firmes françaises en RT, ainsi que par les possibilités croissantes des professionnels tchèques à se présenter sur le marché du travail français.

\section{Bibliographie}

Binon, Serge - Verlinde, Serge - VAn Dyck, Jan - Bertels, Ann (2000), Dictionnaire d'apprentissage du français des affaires, Paris : Didier.

Code De COMmerce (2010), Paris : Dalloz (105e éd.)

Connu, Gérard (1990), Linguistique juridique, Paris : Montchrétien.

Cornu, Gérard (2007), Vocabulaire juridique, Paris : PUF ( $8^{\text {e éd.). }}$

Galisson, Robert (1972), Lexicologie et enseignement des langues, Paris : Hachette.

Kidlesová, Zora - Priesolová, Janka - Brouland, Pierre (2003), Francouzsko-český a česko-francouzský hospodářský slowník, Plzeň : Fraus.

KNAPP, Viktor (1978), "Právní pojmy a právní terminologie », in : Státní správa, Bulletin Ústavu státní správy 4, Praha, p. 4ss.

KnAPP, Viktor (1995), Teorie práva, Praha : C. H. Beck.

LARIŠOVÁ, Markéta (2008), Francouzsko-český a česko-francouzský právnický slovník, Plzeň : A. Čeněk.

Lerat, Pierre (1995), Les langues spécialisées, Paris : PUF.

LeXIQUe Des TERMES JURIDIQues (2009), Paris : Dalloz.

ОвсHODNí ZÁKONíK (2010), ÚZ 769, Sagit.

Priesolové, Janka (2006), « Syntakticko-stylistické problémy odborné francouzštiny v konfrontaci s češtinou », in : Acta Oeconomica Pragensia 4/2006. Ekonomie a cizí jazyky, Praha : Oeconomica, 106-110.

Priesolové, Janka (2004/05), « Nové trendy v moderní lexikografii a jejich přínos pro osvojování ekonomické francouzštiny », Cizí jazyky 48, No 5, 156-159.

ŠABRŠUlA, Jan (2000), Teorie praxe a překladu, Ostrava : Repronis. 
Janka Priesolová

Švarc, Zbyněk et al. (2009), Základy obchodnîho práva, Plzeň : A. Čeněk (2e éd.).

ToMÁšEK, Michal (1998), Překlad v právni praxi, Praha : Linde.

Janka Priesolová

Katedra románských jazyků

Fakulta mezinárodních vztahů

Vysoká škola ekonomická

nám. W. Churchilla 4

13067 Praha 3 - Žižkov

République tchèque

priesolo@vse.cz 